\title{
AN ARITHMETIC PROPERTY OF THE SET OF ANGLES BETWEEN CLOSED GEODESICS ON HYPERBOLIC SURFACES OF FINITE TYPE
}

\author{
SUGATA MONDAL
}

\begin{abstract}
For a hyperbolic surface $S$ of finite type we consider the set $A(S)$ of angles between closed geodesics on $S$. Our main result is that there are only finitely many rational multiples of $\pi$ in $A(S)$.
\end{abstract}

\section{INTRODUCTION}

Geometry of two dimensional manifolds, surfaces, have been in the center of mathematical research for centuries. Hyperbolic metrics on surfaces has been a very important testing ground for different geometric curiosities. In particular, lengths of closed geodesics on these surfaces have been an important topic of research for years (see $[\mathrm{Hu}]$, [O], [W]). This article is on a related geometric quantity the angles between pairs of closed geodesics.

Let $S$ be a hyperbolic surface of finite type. We denote the set of angles between pairs of closed geodesics on $S$ by $\mathcal{A}(S)$. A fixed angle may appear at many different intersections. We call this number of distinct appearances the multiplicity of the angle. We denote the set of angles in $\mathcal{A}(S)$ forgetting their multiplicities by $A(S)$ and call $\mathcal{A}(S)$ and $A(S)$ by angle spectrum and angle set respectively.

We begin by specifying a way of measuring these angles. Let $\gamma$ and $\delta$ be two closed geodesics on $S$ that intersect each other at $p$. We measure the angle of intersection $\theta(\gamma, \delta, p)$ in the counter clockwise direction from $\gamma$ to $\delta$. In particular $\theta(\gamma, \delta, p)=\pi-\theta(\delta, \gamma, p)$.

Remark 1.1. For $\gamma, \delta$ and $\theta=\theta(\gamma, \delta, p)$ as above $\cos ^{2}(\theta)$ depends on $\gamma$ and $\delta$ but not on the direction in which the angle is measured.

In this article we focus on qualitative properties of the two collections $\mathcal{A}(S)$ and $A(S)$. For any hyperbolic surface $S$ of finite type $A(S)$ is a

Date: November 10, 2018.

1991 Mathematics Subject Classification. 53C22, 20H10.

Key words and phrases. Hyperbolic surfaces, Fuchsian groups, angles between geodesics.

Acknowledgments. I would like to thank Chris Judge for the discussions that I had with him on this problem. The example in the last section is due to Hugo Parlier that was explained to the author by Chris Judge. 
countable infinite set and it follows from [P-S that $A(S)$ is dense in $[0, \pi]$. The main question that we address in this paper is the following.

Question 1.2. How many angles in $\mathcal{A}(S)$ can be a rational multiple of $\pi$ ?

Surprisingly the author's motivation to study this question came from a seemingly unrelated field. In the paper [J-M] we have studied eigenfunctions of the Laplacian on hyperbolic surfaces. Let $\phi$ be an eigenfunction of the Laplacian on $\mathbb{H}^{2}$. Let $\Gamma_{\phi}$ denote the subgroup of isometries of $\mathbb{H}^{2}$ that leaves $\phi^{2}$ invariant. In [J-M] we have observed (motivated by a similar observation in [G-R-S]) that if $\phi$ vanishes on a geodesic $\gamma$ then it is odd with respect to the reflection isometry $R_{\gamma}$ along $\gamma$ of $\mathbb{H}^{2}$ i.e. $\phi \circ R_{\gamma}=-\phi$. In particular, $R_{\gamma} \in \Gamma_{\phi}$. An important property of any non-constant eigenfunction $\phi$ is that the subgroup $\Gamma_{\phi}$ is discrete (see $[\mathrm{J}-\mathrm{M}]$ ).

Now consider two intersecting geodesics $\gamma, \delta$ on $\mathbb{H}^{2}$ and consider the subgroup $\Gamma(\gamma, \delta)$ of $\operatorname{SL}(2, \mathbb{R})$ generated by the reflections $R_{\gamma}, R_{\delta}$ along $\gamma$ and $\delta$ respectively. Let $\gamma, \delta$ intersect each other at $p$ and let $\theta=$ $\theta(\gamma, \delta, p)$. Then $\Gamma(\gamma, \delta)$ contains an elliptic isometry of $\mathbb{H}^{2}$ which is a rotation about $p$ by an angle equal to $\theta$. Let $\phi$ be a non-constant eigenfunction that vanish on both $\gamma$ and $\delta$. Then by the last paragraph $\Gamma(\gamma, \delta) \subset \Gamma_{\phi}$. In particular, since $\Gamma_{\phi}$ is discrete, so is $\Gamma(\gamma, \delta)$ implying that $\theta$ must be a rational multiple of $\pi$.

It is not difficult to construct eigenfunctions that vanish on two intersecting geodesics, even on closed hyperbolic surfaces (see [J-M]). In general the answer to Question 1.2 is 'infinite'. In the last section we construct examples of surfaces for which there are infinitely many distinct intersections between pairs of closed geodesics such that the angle of intersection is $\pi / 2$. The main result of this article is that, in general, there are infinitely many rational multiples of $\pi$ in $\mathcal{A}(S)$ if and only if one of these rational multiples of $\pi$ has infinite multiplicity in $\mathcal{A}(S)$.

Theorem 1.3. For any hyperbolic surface $S$ of finite type there are only finitely many angles in $A(S)$ that are rational multiples of $\pi$.

1.1. Structure of the article. In the first section we consider a complete hyperbolic surface $S$ of finite type. Using uniformization theorem we consider a Fuchsian group $\Gamma$ such that $S=\mathbb{H}^{2} / \Gamma$, up to isometry. For two intersecting closed geodesics $\gamma$ and $\delta$ on $S$ we fix an intersection point $p$. In $\S 1$ we consider $M_{\gamma}, M_{\delta} \in \Gamma$ representing $\gamma$ and $\delta$ respectively and use the matrix entries of $M_{\gamma}$ and $M_{\delta}$ to get a formula for $\cos ^{2}(\theta)$ where $\theta=\theta(\gamma, \delta, p)$.

We prove Theorem 1.3 in $\S 2$. In the first step of the proof we consider the field $\mathbb{F}_{\Gamma}$ obtained by attaching the matrix entries of all the elements in $\Gamma$ to $\mathbb{Q}$. Using the fact that $\Gamma$ is finitely generated it follows that $\mathbb{F}_{\Gamma}$ is a finitely generated field extension of $\mathbb{Q}$. Using the expression 
for $\cos ^{2}(\theta)$ obtained in $\S 1$ we deduce that $\cos ^{2}(\theta) \in \mathbb{F}_{\Gamma}$ for any angle $\theta \in A\left(\mathbb{H}^{2} / \Gamma\right)$.

The final arguments of the proof go follows. For simplicity, assume that $\mathbb{F}_{\Gamma}$ is algebraic over $\mathbb{Q}$. Since $\mathbb{F}_{\Gamma}$ is finitely generated over $\mathbb{Q}$ we obtain that the degree of extension $\left.\mathbb{F}_{\Gamma}\right|_{\mathbb{Q}}$ is finite. Now let $\frac{p}{q} \pi$ be in $A\left(\mathbb{H}^{2} / \Gamma\right)$ and so $\cos ^{2}\left(\frac{p}{q} \pi\right) \in \mathbb{F}_{\Gamma}$. Then there is a field extension $\mathbb{F}(q)$ of $\mathbb{F}_{\Gamma}$ with degree of extension at most two that contain a primitive $q$-th root of unity. In particular, the degree of extension $\left.\mathbb{F}(q)\right|_{\mathbb{Q}}$ is uniformly bounded independent of $q$. Finally we observe that the degree of extension $\left.\mathbb{F}(q)\right|_{\mathbb{Q}}$ is at least $\phi(q)$ where $\phi$ is the Euler's $\phi$-function that counts the number of distinct positive integers less than and co-prime with $q$. Since $\phi(q)$ goes to infinity as $q$ goes to infinity [H-W], Theorem $328]$, we reach our desired contradiction.

\section{Formula For the COSINE OF AN ANGLE}

Let $\Gamma$ be a finitely generated Fuchsian group. This usually means that $\Gamma \subset \operatorname{PSL}(2, \mathbb{R})$. By taking the pre-image of $\Gamma$ under the quotient map $\Pi: \operatorname{SL}(2, \mathbb{R}) \rightarrow \operatorname{PSL}(2, \mathbb{R})$ we can always think of $\Gamma \subset \mathrm{SL}(2, \mathbb{R})$. This identification will be assumed in the article from now on. It is a standard fact that every closed geodesic on $S$ corresponds to a conjugacy class of elements in $\pi_{1}(S)=\Gamma$. Let $\gamma, \delta$ be two closed geodesics on $S$ and let $M_{\gamma}, M_{\delta} \in \Gamma$ be two representatives of $\gamma, \delta$ respectively. Let us denote

$$
M_{\gamma}=\left(\begin{array}{cc}
a_{\gamma} & b_{\gamma} \\
c_{\gamma} & d_{\gamma}
\end{array}\right), M_{\delta}=\left(\begin{array}{cc}
a_{\delta} & b_{\delta} \\
c_{\delta} & d_{\delta}
\end{array}\right) .
$$

Recall that $\gamma$ and $\delta$ are the projections of the axes of $M_{\gamma}$ and $M_{\delta}$ respectively, under the covering map: $\mathbb{H}^{2} \rightarrow \mathbb{H}^{2} / \Gamma$. Since $\gamma$ and $\delta$ are closed geodesics, $M_{\gamma}$ and $M_{\delta}$ are hyperbolic linear fractional transformations. Thus the axes of $M_{\gamma}$ and $M_{\delta}$ are either semi-circles or vertical straight lines that intersect $\mathbb{R}$ orthogonally. Here $\mathbb{R} \cup\{\infty\}$ is identified with the boundary $\partial \mathbb{H}^{2}$ of $\mathbb{H}^{2}$.

Observe that in both the cases it is possible to determine the axis of $M_{\gamma}\left(\right.$ or $\left.M_{\delta}\right)$ from the points where they intersect $\mathbb{R}$. Now these last set of points are just the fixed points of $M_{\gamma}$ (or $M_{\delta}$ ). The fixed points of $M_{\gamma}$ can be computed simply as follows. There are two cases.

Case I: First let the axis of $M_{\gamma}\left(\right.$ or $\left.M_{\delta}\right)$ be a semi-circle. Then both the points of intersections are finite real numbers that satisfy

$$
M_{\gamma}(z)=z \Rightarrow \frac{a_{\gamma} z+b_{\gamma}}{c_{\gamma} z+d_{\gamma}}=z \Rightarrow c_{\gamma} z^{2}+\left(d_{\gamma}-a_{\gamma}\right) z-b_{\gamma}=0 .
$$

Hence the two points of intersections of the axis of $M_{\gamma}$ with the real line are the two roots of the equation

$$
c_{\gamma} z^{2}+\left(d_{\gamma}-a_{\gamma}\right) z-b_{\gamma}=0
$$


Denote these by $\alpha_{\gamma}$ and $\beta_{\gamma}$ with $\alpha_{\gamma}<\beta_{\gamma}$. In terms of matrix coefficients of $M_{\gamma}$ we have

$$
\begin{aligned}
& \alpha_{\gamma}=\frac{\left(a_{\gamma}-d_{\gamma}\right)-\sqrt{\left(a_{\gamma}-d_{\gamma}\right)^{2}+4 c_{\gamma} b_{\gamma}}}{2 c_{\gamma}}, \\
& \beta_{\gamma}=\frac{\left(a_{\gamma}-d_{\gamma}\right)+\sqrt{\left(a_{\gamma}-d_{\gamma}\right)^{2}+4 c_{\gamma} b_{\gamma}}}{2 c_{\gamma}} .
\end{aligned}
$$

Using $\operatorname{det} M_{\gamma}=1$ they take the form:

$$
\alpha_{\gamma}=\frac{\left(a_{\gamma}-d_{\gamma}\right)-\sqrt{\operatorname{tr} M_{\gamma}^{2}-4}}{2 c_{\gamma}}, \beta_{\gamma}=\frac{\left(a_{\gamma}-d_{\gamma}\right)+\sqrt{\operatorname{tr} M_{\gamma}^{2}-4}}{2 c_{\gamma}} .
$$

In particular the center and the Euclidean radius of the axis of $M_{\gamma}$ are respectively

$$
m_{\gamma}=\left(\frac{a_{\gamma}-d_{\gamma}}{2 c_{\gamma}}, 0\right) \text { and } r_{\gamma}=\frac{\sqrt{\operatorname{tr} M_{\gamma}^{2}-4}}{2 c_{\gamma}} .
$$

Case II: The axis of $M_{\gamma}$ is a vertical straight line. In particular $c_{\gamma}=0$. Then the only point of intersection between the axis of $M_{\gamma}$ and $\mathbb{R}$ is $\left(\frac{b_{\gamma}}{d_{\gamma}-a_{\gamma}}, 0\right)$.

2.1. Cosine of the angle. Consider two intersecting closed geodesics $\gamma$ and $\delta$ on $S$. Fix one point of their intersection $p$. Choose two representatives $M_{\gamma}, M_{\delta}$ for $\gamma, \delta$ respectively such that the point of intersection $\tilde{p}$ between the axis of $M_{\gamma}$ and the axis of $M_{\delta}$ is a lift of $p$ under the covering map $\pi: \mathbb{H}^{2} \rightarrow \mathbb{H}^{2} / \Gamma=S$. Let $\theta=\theta(\gamma, \delta, p)$. Hence $\theta$ is the angle between the axis of $M_{\gamma}$ and the axis of $M_{\delta}$ at $\tilde{p}$. Now we have two cases depending on the nature of the axes of $M_{\gamma}$ and $M_{\delta}$. We treat them separately.

Case I: First let us assume that both $M_{\gamma}$ and $M_{\delta}$ have semi-circle axes. This situation is explained in the top picture in 1 . Let $\psi$ be the angle between the normals to the the axis of $M_{\gamma}$ and the axis of $M_{\delta}$ at $\tilde{p}$. Then $\psi=\pi-\theta$.

Now consider the Euclidean triangle on $\mathbb{H}^{2}$ with the following three vertices: the centre of the (semi-circle) axis of $M_{\gamma}$, the centre of the (semi-circle) axis of $M_{\delta}$ and $\tilde{p}$ the point of intersection of the two axes. Let us denote the distance between the two centres by $d_{\gamma, \delta}$. Hence

$$
d_{\gamma, \delta}=\left|\frac{a_{\gamma}-d_{\gamma}}{2 c_{\gamma}}-\frac{a_{\delta}-d_{\delta}}{2 c_{\delta}}\right| .
$$

Using Euclidean geometry for the above described triangle we obtain

$$
\cos (\pi-\theta)=\frac{r_{\gamma}^{2}+r_{\delta}^{2}-d_{\gamma, \delta}^{2}}{2 r_{\gamma} r_{\delta}}
$$


Thus

$$
\cos ^{2}(\theta)=\frac{\left(r_{\gamma}^{2}+r_{\delta}^{2}-d_{\gamma, \delta}^{2}\right)^{2}}{4 r_{\gamma}^{2} r_{\delta}^{2}} .
$$

Case II: Now we assume that the axis of $M_{\gamma}$ is a vertical straight line. Since $\gamma$ and $\delta$ intersect each other, the axis of $M_{\delta}$ must be a semi-circle. This situation is explained in the bottom picture of 1 . Consider the normal $N_{\delta}$ to the axis of $M_{\delta}$ at $\tilde{p}$. Let $\psi$ be the angle between the boundary $\partial \mathbb{H}^{2}=\mathbb{R}$ and $N_{\delta}$. Observe that $\psi=\theta$. Now we consider the Euclidean triangle with vertices: the center of the axis of $M_{\delta}$, the point of intersection between axis of $M_{\gamma}$ and $\partial \mathbb{H}^{2}=\mathbb{R}$ and $\tilde{p}$. By the definition of the cosine function and the last equality $\psi=\theta$ we get

$$
\cos ^{2}(\theta)=\left(\frac{\left|\frac{b_{\gamma}}{d_{\gamma}-a_{\gamma}}-\frac{a_{\delta}-d_{\delta}}{2 c_{\delta}}\right|}{\frac{\sqrt{\operatorname{tr} M_{\delta}^{2}-4}}{2 c_{\delta}}}\right)^{2} .
$$
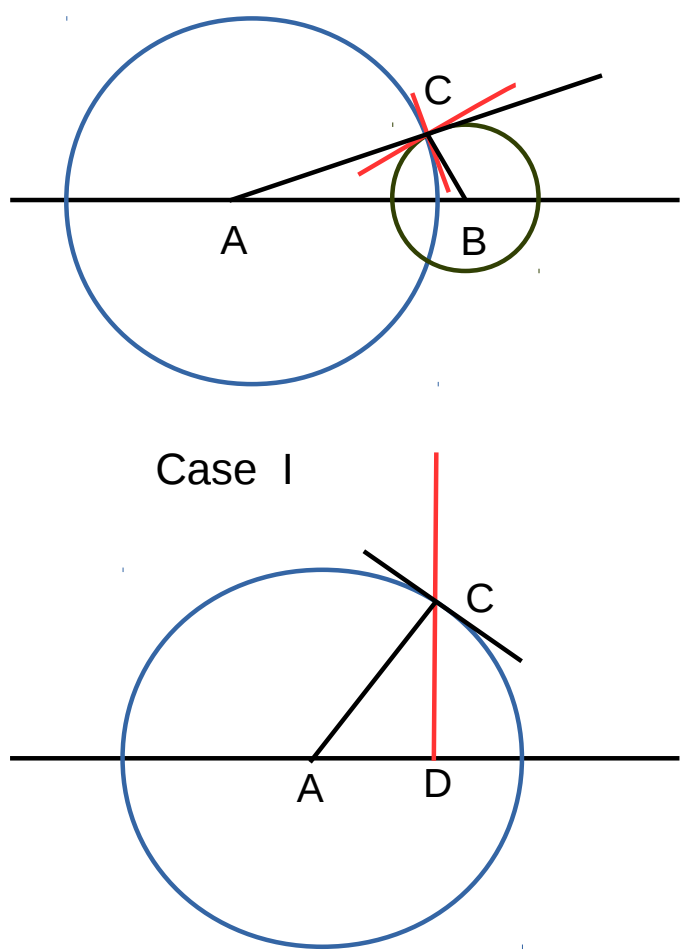

Case II

Figure 1. Cosine of the angle 
Remark 2.8. From the above two expressions it is clear that $\cos ^{2}(\theta)$ is expressible as rational functions in the matrix entries $a_{\gamma}, b_{\gamma}, c_{\gamma}, d_{\gamma}$ of $M_{\gamma}$ and $a_{\delta}, b_{\delta}, c_{\delta}, d_{\delta}$ of $M_{\delta}$.

\section{Proof of Theorem 1.3}

Consider the field

$$
\mathbb{F}_{\Gamma}=\mathbb{Q}\left(a_{\gamma}, b_{\gamma}, c_{\gamma}, d_{\gamma}: M_{\gamma}=\left(\begin{array}{cc}
a_{\gamma} & b_{\gamma} \\
c_{\gamma} & d_{\gamma}
\end{array}\right) \in \Gamma\right)
$$

generated by the entries of the matrices in $\Gamma \subset \mathrm{SL}(2, \mathbb{R})$. Observe that this is a finitely generated field. This is clear because $\Gamma$ is a finitely generated group and so adjoining the matrix entries of a generating subset of $\Gamma$ is enough.

Now we have two cases. The filed $\mathbb{F}_{\Gamma}$ may or may not be algebraic over $\mathbb{Q}$. Let us assume that $\mathbb{F}_{\Gamma}$ is not algebraic over $\mathbb{Q}$. Since $\mathbb{F}_{\Gamma}$ is finitely generated over $\mathbb{Q}$, there is a purely transcendental extension $\left.\mathbb{T}_{\Gamma}\right|_{\mathbb{Q}} \subset \mathbb{F}_{\Gamma}$ such that $\mathbb{F}_{\Gamma}$ is algebraic over $\mathbb{T}_{\Gamma}$. To treat the two cases at the same time let $\mathbb{T}_{\Gamma}$ denote $\mathbb{Q}$ when $\mathbb{F}_{\Gamma}$ is algebraic over $\mathbb{Q}$. In both the cases $\mathbb{F}_{\Gamma}$ is finitely generated over $\mathbb{T}_{\Gamma}$. Hence the degree $\left[\mathbb{F}_{\Gamma}: \mathbb{T}_{\Gamma}\right]$ of the extension $\left.\mathbb{F}_{\Gamma}\right|_{\mathbb{T}_{\Gamma}}$ is finite.

Now from (2.6) and (2.7) it is clear that for $\theta=\theta(\gamma, \delta, p)$ the values $\cos ^{2}(\theta) \in \mathbb{F}_{\Gamma}$. Hence the degree of the field extension $\mathbb{F}_{\Gamma}\left(e^{2 i \theta}\right)_{\mid \mathbb{F}_{\Gamma}}$

$$
\left[\mathbb{F}_{\Gamma}\left(e^{2 i \theta}\right): \mathbb{F}_{\Gamma}\right] \leq 2
$$

This implies that the degree of the extension $\mathbb{T}_{\Gamma}\left(e^{2 i \theta}\right) \mid \mathbb{T}_{\Gamma}$ $\left[\mathbb{T}_{\Gamma}\left(e^{2 i \theta}\right): \mathbb{T}_{\Gamma}\right] \leq\left[\mathbb{F}_{\Gamma}\left(e^{2 i \theta}\right): \mathbb{T}_{\Gamma}\right]=\left[\mathbb{F}_{\Gamma}\left(e^{2 i \theta}\right): \mathbb{F}_{\Gamma}\right] \cdot\left[\mathbb{F}_{\Gamma}: \mathbb{T}_{\Gamma}\right] \leq 2\left[\mathbb{F}_{\Gamma}: \mathbb{T}_{\Gamma}\right]$

Now recall that $\mathbb{T}_{\Gamma}$ is a purely transcendental extension of $\mathbb{Q}$ and so for $\theta$ rational multiple of $\pi$ ( since $e^{2 i \theta}$ is algebraic over $\mathbb{Q}$ ) we always have

$$
\left[\mathbb{T}_{\Gamma}\left(e^{2 i \theta}\right): \mathbb{T}_{\Gamma}\right]=\left[\mathbb{Q}\left(e^{2 i \theta}\right): \mathbb{Q}\right] .
$$

Now let $\theta=\frac{p}{q} \pi$. It is a know fact that the degree $\left[\mathbb{Q}\left(e^{2 i \theta}\right): \mathbb{Q}\right]=\phi(q)$ where $\phi$ is the Euler $\phi$-function. Thus combining the above inequalities we have

$$
\phi(q) \leq 2\left[\mathbb{F}_{\Gamma}: \mathbb{T}_{\Gamma}\right]
$$

Hence there are only finitely many choices for $q$ by $\mathrm{H}-\mathrm{W}$, Theorem 328].

Remarks 3.1. (i) Observe that the field $\mathbb{F}_{\Gamma}$ depends explicitly on $\Gamma$ where $A\left(\mathbb{H}^{2} / \Gamma\right)$ depends only on the conjugacy class of $\Gamma$ because conjugate groups produce isometric surfaces. Hence we conclude that for any $\frac{p}{q} \cdot \pi \in A\left(\mathbb{H}^{2} / \Gamma\right)$

$$
\phi(q) \leq 2 \cdot \min _{\gamma \in \operatorname{PSL}(2, \mathbb{R})}\left[\mathbb{F}_{\gamma \Gamma \gamma^{-1}}, \mathbb{T}_{\gamma \Gamma \gamma^{-1}}\right] .
$$

This can be used to give an explicit bound on the size of $A\left(\mathbb{H}^{2} / \Gamma\right) \cap \mathbb{Q} \cdot \pi$. 
(ii) For the modular surface $\mathbb{H}^{2} / \operatorname{PSL}(2, \mathbb{Z})$ the group $\Gamma$ is $\operatorname{PSL}(2, \mathbb{Z})$ and so the field $\mathbb{F}_{\Gamma}$ is just $\mathbb{Q}$. Hence for any $\frac{p}{q} \pi \in A\left(\mathbb{H}^{2} / \operatorname{PSL}(2, \mathbb{Z})\right)$ we have $\phi(q) \leq 2$ i.e. $q \leq 6$. A simple computation provides that the possible angles are $\pi / 6, \pi / 4$ and $\pi / 3$.

\section{Some QUestions And EXAMPles}

Let $\Gamma$ be a Fuchsian group as above and $S=\mathbb{H}^{2} / \Gamma$. Given an angle $\theta \in A(S)$ one may consider the map $\Theta: A(S) \rightarrow \mathbb{F}_{\Gamma}^{1}$ given by $\Theta(\theta)=\cos ^{2}(\theta)$, where $\mathbb{F}_{\Gamma}^{1} \subset \mathbb{F}_{\Gamma}$ is the set of elements with norm $<1$.

Question 4.1. What is the image of this map ?

Since $A(S)$ is dense in $[0, \pi]$ the image is dense in $[-1,1]$ and hence in $\mathbb{F}_{\Gamma}^{1}$. It is not clear if it equals $\mathbb{F}_{\Gamma}^{1}$ though.

4.1. An angle with infinite multiplicity. Now we show that there are closed hyperbolic surfaces $S$ such that $\pi / 2$ has infinite multiplicity in $\mathcal{A}(S)$. To construct such a surface start with a compact surface $S^{\prime}$ with geodesic boundary and consider its double $D S^{\prime}$. Observe that $D S^{\prime}$ has a reflection isometry along the boundary geodesics of $S^{\prime}$. Now take a closed geodesic that is symmetric with respect to this reflection and intersects at least one of the boundary geodesics of $S^{\prime}$. By the reflection symmetry each of these angles of intersection must be equal to $\pi / 2$. It is not hard to construct a surface having infinitely many geodesics of this type. This poses our last question.

Question 4.2. What angles in $\mathcal{A}(S)$ can have infinite multiplicity?

\section{REFERENCES}

[G-R-S] Ghosh, A.; Reznikov, A.; Sarnak, P.; Nodal domains of Maas forms I. Geom. Funct. Anal. Vol. 23 (2013) 1515-1568.

[H-W] Hardy, G. H.; Wright, E. M.; An Introduction to the Theory of Numbers (Fifth ed.), Oxford: Oxford University Press, ISBN 978-0-19-853171-5

[Hu] Huber, H.; Zur analytischen Theorie hyperbolischer Raumformen und Bewegungsgruppen I, II, Nachtrag zu II. Math. Ann. 138 (1959), 1-26. Math. Ann. 142 (1961), 385-398; Math. Ann. 143 (1961), 463-464.

[J-M] Judge, C.; Mondal, S.; Geodesics and Nodal sets of Laplace eigenfunctions on hyperbolic manifolds. To appear in Proc. of the AMS.

[O] Otal, J-P.; Le spectre marqu des longueurs des surfaces courbure ngative. (French) [The marked spectrum of the lengths of surfaces with negative curvature] Ann. of Math. (2) 131 (1990), no. 1, 151162.

[P-S] Pollicott, M.; Sharp, R.; Angular self-intersections for closed geodesics on surfaces. Proc. of the AMS, Volume 134, Number 2, Pages 419-426.

[W] Wolpert, S.; The length spectra as moduli for compact Riemann surfaces. Ann. of Math. (2) 109 (1979), no. 2, 323351.

Indiana University, Rawles Hall, 831 E 3Rd Street, Bloomington, INDIANA,

E-mail address: sumondal@iu.edu 